\title{
An Analysis of a Uniformly Accurate Difference Method for a Singular Perturbation Problem*
}

\author{
By Alan E. Berger, Jay M. Solomon and Melvyn Ciment
}

\begin{abstract}
It will be proven that an exponential tridiagonal difference scheme, when applied with a uniform mesh of size $h$ to: $e u_{x x}+b(x) u_{x}=f(x)$ for $0<x<1, b>0, b$ and $f$ smooth, $\varepsilon$ in $(0,1]$, and $u(0)$ and $u(1)$ given, is uniformly second-order accurate (i.e., the maximum of the errors at the grid points is bounded by $\mathrm{Ch}^{2}$ with the constant $C$ independent of $h$ and $\varepsilon$ ). This scheme was derived by El-Mistikawy and Werle by a $C^{1}$ patching of a pair of piecewise constant coefficient approximate differential equations across a common grid point. The behavior of the approximate solution in between the grid points will be analyzed, and some numerical results will also be given.
\end{abstract}

I. Introduction and Notation. This paper is devoted to the error analysis of a particular three-point finite difference scheme derived by El-Mistikawy and Werle for the solution of the following singular perturbation problem

$$
L u \equiv \varepsilon u_{x x}+b(x) u_{x}-d(x) u=f(x) \text { for } 0<x<1, u(0)=\alpha_{0}, u(1)=\alpha_{1},
$$

where $\varepsilon$ is a parameter in $(0,1] ; \alpha_{0}$ and $\alpha_{1}$ are given constants; $b, d$, and $f$ are in $C^{m}[0,1]$ (throughout this paper $m=5$ ); $d>0$, and $b(x)>B_{1}$ on $[0,1]$ for some positive constant $B_{1}$. Under these assumptions, (1.1) has a unique solution $u$ which in general displays a boundary layer at $x=0$ for "small" $\varepsilon$, e.g., [14], [8]. The problem (1.1) and the associated initial boundary value problem for $L u=u_{t}$ when $\varepsilon$ is small are prototypes of the problems which arise, for example, in the modeling of steady and unsteady viscous flow problems with large Reynolds numbers and convective heat transport problems with large Peclet numbers.

We will consider a particular finite difference method for the numerical solution of (1.1) which is a member of the following family of difference schemes. Let $J$ be a positive integer and define the uniform mesh length $h=1 / J$. Let the grid points $\left\{x_{j}\right\}$ be given by $x_{j}=j h, j=0,1, \ldots, J$, and let $U_{j}$ denote the approximate value (to be determined) for $u_{j}=u\left(x_{j}\right)$. When applied to (1.1), the family of schemes has the form

$$
\varepsilon h^{-2}\left(r_{j}^{-} U_{j-1}+r_{j}^{c} U_{j}+r_{j}^{+} U_{j+1}\right)=q_{j}^{-} f_{j-1}+q_{j}^{c} f_{j}+q_{j}^{+} f_{j+1}
$$

for $j=1, \ldots, J-1$, with

$$
U_{0} \equiv \alpha_{0} \quad \text { and } \quad U_{J} \equiv \alpha_{1}
$$

Received October 27, 1980.

1980 Mathematics Subject Classification. Primary 65L10, 65M05, 65M10; Secondary 34E15.

- This work was supported jointly by the Office of Naval Research (Project No. RR014-03-01) and the NSWC Independent Research Fund and the National Engineering Laboratory Director's Reserve Fund of the National Bureau of Standards. 
Here $f_{j}$ denotes $f\left(x_{j}\right)$ etc. The choice of the coefficients $r_{j}^{-}, r_{j}^{c}, r_{j}^{+}$and $q_{j}^{-}, q_{j}^{c}, q_{j}^{+}$ determines the particular scheme. When it will be clear from the context, the $j$ subscripts in $r_{j}^{-}, \ldots, q_{j}^{+}$will be omitted. It will be convenient to employ the following notation; given an arbitrary set of values $V_{j}$ at the grid points $x_{j}$,

$$
\begin{aligned}
& R V_{j} \equiv r^{-} V_{j-1}+r^{c} V_{j}+r^{+} V_{j+1}, \quad \text { and } R^{h} V_{j} \equiv \varepsilon h^{-2} R V_{j}, \\
& Q V_{j} \equiv q^{-} V_{j-1}+q^{c} V_{j}+q^{+} V_{j+1} .
\end{aligned}
$$

To illustrate this notation, consider, for example, the standard one-sided (upwind) scheme for (1.1) given by

$$
\varepsilon h^{-2}\left(U_{j-1}-2 U_{j}+U_{j+1}\right)+b_{j}\left(U_{j+1}-U_{j}\right) / h-d_{j} U_{j}=f_{j}
$$

For this particular scheme, the $r$ 's and $q$ 's are given by

$$
\begin{array}{ll}
r^{-}=1, & r^{c}=-2-h b_{j} / \varepsilon-h^{2} d_{j} / \varepsilon, \quad r^{+}=1+h b_{j} / \varepsilon, \\
q^{c}=1, & q^{-}=q^{+}=0 .
\end{array}
$$

The scheme for (1.1) to be considered here was derived in [4] and has the following form when $d \neq 0$. Let $\bar{n}_{1}$ denote the negative root of $\varepsilon w^{2}+$ $\left(b_{j-1}+b_{j}\right) w / 2-\left(d_{j-1}+d_{j}\right) / 2=0$ and let $\bar{k}_{1}$ denote the nonnegative root. Define $n_{1}=h \bar{n}_{1}$ and $k_{1}=h \bar{k}_{1}$. Similarly define $n_{2}$ and $k_{2}$ using the quadratic $\varepsilon w^{2}+$ $\left(b_{j}+b_{j+1}\right) w / 2-\left(d_{j}+d_{j+1}\right) / 2$. Define the following functions: $e(w) \equiv \exp (w)$, $g(w) \equiv(e(w)-1) / w$ with $g(0) \equiv 1$, and let $2 v_{1} \equiv\left[1-e\left(n_{1}-k_{1}\right)\right]^{-1}$ and $2 v_{2} \equiv$ $\left[1-e\left(n_{2}-k_{2}\right)\right]^{-1}$. The scheme [4] at point $x_{j}$ then has the form

$$
\begin{aligned}
r^{-} & =e\left(n_{1}\right) / g\left(n_{1}-k_{1}\right), \quad r^{+}=e\left(-k_{2}\right) / g\left(n_{2}-k_{2}\right), \\
r_{1} & =-n_{1}-1 / g\left(n_{1}-k_{1}\right), \quad r_{2}=k_{2}-1 / g\left(n_{2}-k_{2}\right), \\
r^{c} & =r_{1}+r_{2}, \\
q^{-} & =g\left(n_{1}\right) v_{1}-e\left(n_{1}\right) g\left(-k_{1}\right) v_{1}, \\
q^{+} & =g\left(-k_{2}\right) v_{2}-e\left(-k_{2}\right) g\left(n_{2}\right) v_{2}, \quad q^{c}=q^{-}+q^{+} .
\end{aligned}
$$

In the situation where $d \equiv 0$, this scheme reduces to

$$
\begin{aligned}
r^{-} & =\rho^{-} \exp \left(-\rho^{-}\right) /\left[1-\exp \left(-\rho^{-}\right)\right], \quad r^{+}=\rho^{+} /\left[1-\exp \left(-\rho^{+}\right)\right], \\
r^{c} & =-r^{-}-r^{+}, \quad q^{-}=\left(1-r^{-}\right) /\left(2 \rho^{-}\right), \quad q^{+}=\left(r^{+}-1\right) /\left(2 \rho^{+}\right), \\
q^{c} & =q^{-}+q^{+}, \quad \text { where } \rho^{-}=\left(b_{j-1}+b_{j}\right) h /(2 \varepsilon), \\
\rho^{+} & =\left(b_{j}+b_{j+1}\right) h /(2 \varepsilon) .
\end{aligned}
$$

A sketch of the derivation of (1.4) (as done in [4], cf. also the references in [4], particularly [12] and [13]) will be given in Section 2 below.

The principal result of this paper is

THEOREM 1.1. Assume $d \equiv 0$, and let $\left\{U_{j}\right\}$ be the approximate solution of (1.1) obtained by using (1.5). Then there is a constant $C$, depending only on $b, f, \alpha_{0}, \alpha_{1}$, such that for all $\varepsilon$ in $(0,1]$

$$
\left|U_{j}-u\left(x_{j}\right)\right| \leqslant C h^{2} \text { for } j=1, \ldots, J-1 .
$$

The constant $C$ in (1.6) will be seen to depend only on the set $S_{2}$ (with $m=5$ and $B_{9}=\delta_{1}=0$ ) defined in the beginning of Section 3. Our proof of Theorem 1.1, based on the comparison function approach of Kellogg and Tsan [8], was briefly 
outlined in [3] and [2]. An independent proof of Theorem 1.1 has now also been obtained by Hegarty, Miller and O'Riordan [6] using the "double mesh method" (cf. [7], [10]). Lorenz [9] has obtained the result (1.6) for nonzero $d(x)$ for a related scheme under the assumption that $b$ is a positive constant. Finite element methods employing particular exponential type functions in the trial and test spaces were formulated and analyzed in [5].

For a detailed discussion of properties of schemes of the form (1.2) (e.g., maximum principle, cell Reynolds number condition, formal application to $L u=$ $u_{t}$ ) and for a comparison of the theoretical and numerical convergence behavior of (1.5) with that for a number of other schemes for (1.1) see [3], and also [1], [2], and the references therein. Here we proceed directly to presenting the basic properties of (1.4) and then to proving Theorem 1.1. A numerical experiment, whose result is consistent with the conjecture that (1.4) is also uniformly second-order accurate for (1.1), is given in Section 5.

II. Derivation and Basic Properties of (1.4). The schemes (1.4) and (1.5) can be derived as follows [4]. Consider the problem

$$
\bar{L} U \equiv \varepsilon U_{x x}+\bar{b} U_{x}-\bar{d} U=\bar{f} \text { for } x_{j-1}<x<x_{j+1}
$$

with

$$
U\left(x_{j-1}\right) \equiv U_{j-1} \quad \text { and } \quad U\left(x_{j+1}\right) \equiv U_{j+1},
$$

where $U_{j-1}$ and $U_{j+1}$ are regarded, at this point, as prescribed numbers, and where

$$
\bar{b}(x) \equiv \begin{cases}\left(b_{j-1}+b_{j}\right) / 2 & \text { for } x_{j-1}<x<x_{j} \\ \left(b_{j}+b_{j+1}\right) / 2 & \text { for } x_{j}<x<x_{j+1}\end{cases}
$$

and $\bar{d}$ and $\bar{f}$ are similarly defined. This problem has a unique solution $U(x)$ in $C^{1}\left[x_{j-1}, x_{j+1}\right]$; indeed, for any given value $U_{j}$, there are unique solutions $U_{1}(x)$ of $\bar{L} U_{1}=\bar{f}$ on $\left(x_{j-1}, x_{j}\right)$ with $U_{1}\left(x_{j-1}\right)=U_{j-1}$ and $U_{1}\left(x_{j}\right)=U_{j}$; and $U_{2}(x)$ of $\bar{L} U_{2}=\bar{f}$ on $\left(x_{j}, x_{j+1}\right)$ with $U_{2}\left(x_{j}\right)=U_{j}$ and $U_{2}\left(x_{j+1}\right)=U_{j+1}$. With $U_{j-1}$ and $U_{j+1}$ considered as given, there is then a unique choice of $U_{j}$ for which it is true that

$$
D_{x} U_{1}\left(x_{j}\right)=D_{x} U_{2}\left(x_{j}\right) \text {, }
$$

where $D_{x}^{k}$ denotes the operation of taking $k x$-derivatives and $D_{x} \equiv D_{x}^{1}$. The equation (2.2), when written out, produces (1.4) (or (1.5) when $d \equiv 0$ ). Note that, as $d \rightarrow 0$, (1.4) becomes (1.5). The above derivation of (1.4) beginning with (2.1) is also valid in the situation where $d>0$ on $\left[x_{j-1}, x_{j+1}\right]$ and $b(x)$ is an arbitrary function on $\left[x_{j-1}, x_{j+1}\right]$ (e.g., $b(x)$ being allowed to go through zero).

It will be convenient to have the following observation for use in verifying properties of the $r$ 's and $q$ 's.

Remark 2.1. Let $g(x)$ be a smooth function satisfying $g(0)=D_{x} g(0)=\ldots=$ $D_{x}^{k} g(0)=0$ and $D_{x}^{k+1} g(x)>0$ for $x>0$ ( $k$ some nonnegative integer). Then $g>0$ for $x>0$.

Using Remark 2.1 and some algebra, one can verify

Remark 2.2. Consider the formulas (1.4) as algebraic formulas independent of their origin, with only the assumptions that $n_{1}<0, n_{2}<0, k_{1}>0$, and $k_{2}>0$. 
Then it is true that

$$
r^{-}>0, \quad r^{+}>0, \quad-r_{1}>r^{-}, \quad-r_{2}>r^{+}, \quad q^{-}>0, q^{+}>0 .
$$

For example, to show $-r_{1} \geqslant r^{-}$, take out the common denominator $\exp \left(n_{1}-k_{1}\right)$ -1 and then verify the result for each fixed $n_{1}<0$ using the approach of Remark 2.1 on the resulting function of $k_{1}$. One thus finds it suffices to show $1-\exp \left(n_{1}\right)+$ $n_{1} \exp \left(n_{1}\right)>0$ for $n_{1}<0$, and the idea of Remark 2.1 again applies.

As pointed out in Section 2.2 of [3], (2.3) implies that the linear system of equations given by $(1.2 \mathrm{a}, \mathrm{b})$ can be solved by simple tridiagonal Gaussian decomposition and that (1.4) satisfies a discrete maximum principle, i.e.,

Suppose $\left\{V_{j}\right\}$ is a set of values at the grid points $x_{j}$ satisfying

$V_{0}<0, \quad V_{J}<0$, and $R V_{j}>0$ for $j=1, \ldots, J-1$. Then

$V_{j}<0$ for $j=0,1, \ldots, J$.

This discrete maximum principle permits the use of the comparsion function approach for an error analysis of the scheme [8], [3], [2].

We now turn our attention to the analysis of (1.5) (a comparison function analysis of (1.4) would seem at best to involve an overwhelming amount of algebra). We conclude this section with a collection of technical results on the properties of the $r$ 's and $q$ 's of (1.5) which will be used in the proof of Theorem 1.1. Corresponding to (1.5) we define the following functions of $\rho$ :

$$
\begin{aligned}
& r^{-}(\rho)=\rho \cdot \exp (-\rho) /[1-\exp (-\rho)], \quad r^{-}(0) \equiv 1, \\
& r^{+}(\rho)=\rho /[1-\exp (-\rho)], \quad r^{+}(0) \equiv 1, \quad q^{-}(\rho)=\left(1-r^{-}(\rho)\right) /(2 \rho) \\
& q^{-}(0) \equiv 1 / 4, \quad q^{+}(\rho)=\left(r^{+}(\rho)-1\right) /(2 \rho), \quad q^{+}(0) \equiv 1 / 4
\end{aligned}
$$

Remark 2.3. The functions $r^{-}, r^{+}, q^{-}, q^{+}$in (2.5) are $C^{\infty}$ on $R^{1} . D_{\rho} r^{-}(0)=$ $-1 / 2, D_{\rho} r^{-}<0$ on $0<\rho<\infty$, and $D_{\rho} r^{-} \rightarrow 0$ as $\rho \rightarrow \infty$. $D_{\rho} r^{+}(0)=1 / 2, D_{\rho} r^{+}$ $>0$ on $0<\rho<\infty$, and $D_{\rho} r^{+} \rightarrow 1$ as $\rho \rightarrow \infty \cdot q^{-}(\rho)>0$ on $0<\rho<\infty$ and $q^{-} \rightarrow 0$ as $\rho \rightarrow \infty \cdot q^{+}(\rho)>0$ on $0<\rho<\infty$ and $q^{+} \rightarrow 1 / 2$ as $\rho \rightarrow \infty$.

Remark 2.4. The following power series expansions about $\rho=0$ obtain;

$$
\begin{aligned}
& r^{-}(\rho)=1-\rho / 2+\rho^{2} / 12+O\left(\rho^{4}\right), \\
& r^{+}(\rho)=1+\rho / 2+\rho^{2} / 12+O\left(\rho^{4}\right), \\
& q^{-}(\rho)=1 / 4-\rho / 24+O\left(\rho^{3}\right), \\
& q^{+}(\rho)=1 / 4+\rho / 24+O\left(\rho^{3}\right) .
\end{aligned}
$$

Remark 2.5. $r^{+}(\rho)-r^{-}(\rho)=\rho$. Also, let $C_{1}$ be a given positive constant. Then there are constants $C_{2}$ and $C_{3}$, depending only on $C_{1}$, such that for $\rho>C_{1}$ it is true that

$$
\begin{aligned}
D_{\rho} r^{-}(\rho) & =\exp (-\rho)(1-\rho-\exp (-\rho)) /(1-\exp (-\rho))^{2}, \\
\left|D_{\rho} r^{-}(\rho)\right| & <C_{2} \exp (-\rho / 2), \quad\left|D_{\rho} r^{-}(\rho)\right|<C_{2} \rho \cdot \exp (-\rho), \\
D_{\rho} r^{+}(\rho) & <C_{2}, \quad\left|D_{\rho} q^{-}(\rho)\right|+\left|D_{\rho} q^{+}(\rho)\right|<C_{3} \rho^{-2}, \\
\left|D_{\rho}^{2} r^{-}(\rho)\right| & <C_{2} \exp (-\rho / 2) .
\end{aligned}
$$


III. Comparison Function Proof of Theorem 1.1. A detailed overview of the comparison function approach as employed by Kellogg and Tsan [8] has been given (in terms of our notation) in [3], and we shall directly proceed with the proof following the outline given in Section 4.2 of [3]. We first need to introduce the hypotheses and some notation. Let $B_{1}$ be a positive constant such that $b(x)>B_{1}$ on $[0,1]$, and define $B_{5}$ to be the norm of $b(x)$ in $C^{m}[0,1]$ (for Theorem 1.1 $m \equiv 5)$. To accomplish our proof, we allow the function $f$ in (1.1) to depend on $x$ and $\varepsilon$ such that $f(x, \varepsilon)$ has $m$ continuous $x$-derivatives satisfying

$$
\left|f^{(i)}(x, \varepsilon)\right|<B_{7}+B_{9} \varepsilon^{-i} \exp \left(-\delta_{1} x / \varepsilon\right) \text { for } i=0,1, \ldots, m,
$$

$x$ in $[0,1]$, and $\varepsilon$ in $(0,1]$, where $B_{7}, B_{9}$, and $\delta_{1}$, are positive constants (independent of $x$ and $\varepsilon$ ).

Note, however, that for Theorem 1.1 it is assumed that $f$ is independent of $\varepsilon$ (in which case $\left.B_{9} \equiv \delta_{1} \equiv 0\right)$. Let $B_{8}=\left|\alpha_{0}\right|+\left|\alpha_{1}\right|$, and let $S_{2}$ denote the set $\left\{B_{1}, B_{5}, B_{7}, \delta_{1}, B_{8}, B_{9}\right\}$. Throughout the rest of this paper $c, C, c_{i}, C_{i}(i=0,1, \ldots)$ will be used to denote generic positive constants which may depend on elements of the set $S_{2}$ but which are independent of $h$ and $\varepsilon$.

We will prove below the following preliminary error estimate.

Theorem 3.1. Assume $d=0$ in (1.1) and let $\left\{U_{j}\right\}, j=0, \ldots, J$, be the approximation to the solution $u(x)$ of (1.1) obtained using (1.5). Assume the hypotheses of this section are valid with $m=3$. Then there are constants $\sigma$ and $C$, depending only on $S_{2}$, such that for $j=1, \ldots, J-1$,

$$
\begin{aligned}
& \left|U_{j}-u\left(x_{j}\right)\right| \leqslant C h^{2}+C h^{2} \varepsilon^{-1} \exp \left(-\sigma x_{j} / \varepsilon\right) \text { when } h<\varepsilon, \\
& \left|U_{j}-u\left(x_{j}\right)\right|<C h^{2}+C \varepsilon \cdot \exp \left(-\sigma x_{j-1} / \varepsilon\right) \text { when } \varepsilon<h .
\end{aligned}
$$

This result, along with the following expansion of the solution $u(x)$ of (1.1) will enable us to demonstrate Theorem 1.1.

Lemma 3.2 (Sмith [14, pp. 253-258]). Assume the hypotheses of this section are satisfied with $m=5$, and assume $f(x)$ is independent of $\varepsilon\left(\right.$ so $\left.B_{9} \equiv \delta_{1} \equiv 0\right)$. Then the solution $u(x)$ of (1.1) can be written in the form

$$
u(x)=A_{0}(x)+C_{0} E(x) / b(x)+\varepsilon R_{0}(x)
$$

where

$$
E(x) \equiv \exp \left(-\varepsilon^{-1} \int_{0}^{x} b(\xi) d \xi\right),
$$

and where the magnitude of the constant $C_{0}$ and the norm of $A_{0}(x)$ in $C^{4}[0,1]$ depend only on $S_{2}$. The function $R_{0}(x)$ satisfies the equation

$$
\varepsilon r_{x x}+b(x) r_{x}=F_{0}(x, \varepsilon) \text { for } 0<x<1, r(0)=0, r(1)=\gamma_{0}(\varepsilon)
$$

where, for $\varepsilon$ in $(0,1],\left|\gamma_{0}(\varepsilon)\right|$ has an upper bound depending only on $S_{2}$, and where $F_{0}(x, \varepsilon)$ satisfies the hypothesis of Theorem $3.1\left(B_{7}, B_{9}\right.$, and $\delta_{1}$ for $F_{0}$ depend only on the set $S_{2}$ from (1.1)).

Thus Theorem 3.1 implies that the contribution to the total error from using (1.5) on the $\varepsilon R_{0}(x)$ summand is uniformly $O\left(h^{2}\right)$ (i.e., bounded by $C h^{2}$ ). The contribution from the other two summands in (3.3a) will be shown below to be also uniformly $O\left(h^{2}\right)$, which will then complete the proof of Theorem 1.1. 
The following two lemmas bound the behavior of the solution $u(x)$ of $(1.1)$ (with $f$ satisfying (3.1)) and are used in the comparison function proof to bound the truncation error.

LeMma 3.3 (KellogG AND TSAN [8]). Equation (1.1) has a unique solution, and there are positive constants $\delta$ and $C$, depending only on $S_{2}$, such that

$$
\left|u^{(i)}(x)\right|<C+C \varepsilon^{-i} \exp (-2 \delta x / \varepsilon) \text { for } i=0,1, \ldots, m+1 .
$$

Here and in Lemma $3.4 m$ is that of (3.1).

LEMMA 3.4 (slight extension of Lemma 2.4 of [8]). The solution of (1.1) can be written in the form

$$
u(x)=\left(-\varepsilon u_{x}(0) / b(0)\right) \exp (-b(0) x / \varepsilon)+w(x),
$$

where, for some positive constants $\delta$ and $C$ depending only on $S_{2}$,

$$
\left|w^{(i)}(x)\right| \leqslant C\left[1+\varepsilon^{-i+1} \exp (-2 \delta x / \varepsilon)\right] \text { for } i=0,1, \ldots, m+1 .
$$

The two comparison functions to be used are

$$
\varphi_{j} \equiv-2+x_{j} \quad \text { and } \quad \psi_{j}=\psi_{j}(\beta) \equiv-[\mu(\beta)]^{j},
$$

where $\mu(\beta) \equiv r^{-}(\beta h / \varepsilon) / r^{+}(\beta h / \varepsilon)=\exp (-\beta h / \varepsilon)$ for some $\beta>0$ to be chosen ( $\beta$ will be taken to be the smallest of various positive constants appearing in the proof).

3.1. The Truncation Error for (1.5). The truncation error of the scheme is defined to be

$$
\tau_{j}=R^{h} u\left(x_{j}\right)-Q\left(L u\left(x_{j}\right)\right) \text { for } j=1, \ldots, J-1 .
$$

For $u(x)$ sufficiently smooth, the standard formal Taylor development of $\tau_{j}$ for $\varepsilon$ fixed has the form

$$
\tau_{j}=\tau_{j}(u)=T^{0}(R, Q, h, \varepsilon, j) u\left(x_{j}\right)+T^{1}(R, Q, h, \varepsilon, j) u^{(1)}\left(x_{j}\right)+\ldots
$$

The specific form of $T^{0}, T^{1}, \ldots, T^{6}$ is given in Eq. (2.5) of [3]. As in [8], when $\varepsilon<h$ essential use will be made of the integral form of the remainder in Taylor's theorem; viz., for a sufficiently smooth function $g(x)$ and numbers $a$ and $p$,

$$
\begin{aligned}
R_{n}(a, p, g) & \equiv g(p)-\sum_{i=0}^{n} g^{(i)}(a) \frac{(p-a)^{i}}{i !}=g^{(n+1)}(\xi) \frac{(p-a)^{n+1}}{(n+1) !} \\
& =\frac{1}{n !} \int_{a}^{p}(p-s)^{n} g^{(n+1)}(s) d s .
\end{aligned}
$$

Here $\xi$ is a point between the points $a$ and $p$. Once can easily verify that, for the scheme (1.5), $T^{0}$ and $T^{1}$ from (3.7) are 0 .

Expanding out to $u^{(4)}(x)$ terms (which is the appropriate expansion for $h<\varepsilon$ ), the truncation error has the form

$$
\begin{aligned}
\tau_{j}= & T^{2} u^{(2)}\left(x_{j}\right)+T^{3} u^{(3)}\left(x_{j}\right)+r^{-} \varepsilon h^{-2} R_{3}\left(x_{j}, x_{j}-h, u\right) \\
& +r^{+} \varepsilon h^{-2} R_{3}\left(x_{j}, x_{j}+h, u\right)-q^{-} \varepsilon R_{1}\left(x_{j}, x_{j}-h, u_{x x}\right) \\
& -q^{-} b_{j-1} R_{2}\left(x_{j}, x_{j}-h, u_{x}\right)-\varepsilon q^{+} R_{1}\left(x_{j}, x_{j}+h, u_{x x}\right) \\
& -q^{+} b_{j+1} R_{2}\left(x_{j}, x_{j}+h, u_{x}\right),
\end{aligned}
$$


where

$$
\begin{aligned}
& T^{2}=\left(r^{-}+r^{+}\right) \varepsilon / 2+\left(b_{j-1} h-\varepsilon\right) q^{-}-\varepsilon q^{c}-\left(b_{j+1} h+\varepsilon\right) q^{+}, \\
& T^{3}=\varepsilon h\left(r^{+}-r^{-}\right) / 6+\left(\varepsilon h-b_{j-1} h^{2} / 2\right) q^{-}-\left(b_{j+1} h^{2} / 2+\varepsilon h\right) q^{+} .
\end{aligned}
$$

For $h>\varepsilon$, the expansion which is suitable for obtaining the desired error estimate is

$$
\begin{aligned}
\tau_{j}= & T^{2} u^{(2)}\left(x_{j}\right)+r^{-} \varepsilon h^{-2} R_{2}\left(x_{j}, x_{j}-h, u\right) \\
& +r^{+} \varepsilon h^{-2} R_{2}\left(x_{j}, x_{j}+h, u\right)-q^{-} \varepsilon R_{0}\left(x_{j}, x_{j}-h, u_{x x}\right) \\
& -q^{-} b_{j-1} R_{1}\left(x_{j}, x_{j}-h, u_{x}\right)-\varepsilon q^{+} R_{0}\left(x_{j}, x_{j}+h, u_{x x}\right) \\
& -q^{+} b_{j+1} R_{1}\left(x_{j}, x_{j}+h, u_{x}\right),
\end{aligned}
$$

where $T^{2}$ is again given by (3.8b). Note that if $b$ is constant, then $T^{2}$ vanishes.

A first step in obtaining Theorem 1.1 is to show the following.

LEMMA 3.5. Suppose in (3.8) that the first four derivatives of $u$ are uniformly bounded by some constant $C_{u}$ for $0 \leqslant x \leqslant 1$ and $0<\varepsilon<1$. Then $\tau_{j}$ is uniformly $O\left(h^{2}\right)$, i.e, there is a constant $C$, depending only on $S_{2}$ and $C_{u}$, such that $\left|\tau_{j}\right| \leqslant C h^{2}$.

Proof. This follows by inspection and Remark 2.3 for the remainder terms, so it suffices to show that $T^{2}$ and $T^{3}$ are uniformly $O\left(h^{2}\right)$. For $T^{3}$, when $\varepsilon<h$, this is again easily verified, while (2.6) suffices to give the result for $h<\varepsilon$. For $T^{2}$, observe that $r^{-}\left(\rho^{-}\right)=r^{-}\left(\rho^{+}\right)+\left(\rho^{-}-\rho^{+}\right) D_{\rho} r^{-}\left(\xi_{1}\right)$ and similarly for $q^{-}\left(\rho^{-}\right)$. Since $T^{2}$ vanishes for $b$ constant, the result follows for $h<\varepsilon$, and (noting (2.7)) for $\varepsilon<h$ as well.

3.2. Lower Bounds for $R^{h} \varphi_{j}$ and $R^{h} \psi_{j}$. We now obtain lower bounds for $R^{h} \varphi_{j}$ and $R^{h} \psi_{j}$.

LEMMA 3.6. There is a constant c, depending only on $S_{2}$, such that $R^{h} \varphi_{j} \geqslant c$ for $\varepsilon$ in $(0,1]$ and $h<1$.

Proof. Since $T^{0}=T^{1}=0, \tau_{j}$ for $\varphi(x)=-2+x$ is $0=R^{h} \varphi_{j}-Q L \varphi_{j}$, so $R^{h} \varphi_{j}=$ $Q b_{j}$, and the result follows from Remark 2.3.

Note that Lemmas 3.6 and 3.5 , along with the maximum principle (2.4), imply that the contribution to the error from approximating the summand $A_{0}(x)$ in (3.3a) using (1.5) is bounded by $C h^{2}$.

For the rest of the proof of Theorem 1.1, it will be convenient to take $h$ bounded above by some "small" constant (independent of $\varepsilon$ ). This is permissible by Remark 4.16 of [3]. We now turn our attention to finding a lower bound for $R^{h} \psi_{j}$.

LEMMA 3.6. There exist constants $c_{1}$ and $c_{2}$, depending only on $S_{2}$, such that when $h \leqslant c_{1}$ and $0<\beta \leqslant c_{2}$, then, for $j=1, \ldots, J-1$, and for some constant $C_{\beta}$ depending only on $S_{2}$ and $\beta$, it is true that

$$
\begin{aligned}
R^{h} \psi_{j}(\beta) & \geqslant C_{\beta} \mu^{j}(\beta) / \varepsilon & \text { for } h<\varepsilon, \\
R^{h} \psi_{j}(\beta) & \geqslant C_{\beta} \mu^{j}(\beta) / h & \text { for } h>\varepsilon, \\
R^{h} \psi_{j}(\beta) / \mu(\beta) & \geqslant C_{\beta} \mu^{j-1}(\beta) / h & \text { for } h \geqslant \varepsilon .
\end{aligned}
$$


Proof. One can check that

$$
R^{h} \psi_{j}(\beta)=\varepsilon h^{-2} \mu(\beta)^{j-1} r^{+}(1-\mu)\left(\mu-r^{-} / r^{+}\right) .
$$

The estimate (3.10) is obtained by estimating the individual factors in (3.11) for the three cases (i) $h / \varepsilon \leqslant c$, (ii) $h / \varepsilon \geqslant C$, and (iii) $c<h / \varepsilon<C$ (for appropriately chosen $c$ and $C$ ). Take $c_{2}<\min \left(1, B_{1} / 2\right)$. For (i) and for $c$ sufficiently small: $r^{+} \approx 1,1-\mu>C_{3} \beta h / \varepsilon, \mu(\beta)-r^{-} / r^{+}>c_{4}(\beta) h / \varepsilon$, and (3.10) holds for (i). For (ii) and for $C$ sufficiently large: $r^{+}>B_{1} h / \varepsilon, 1-\mu>C_{5}(\beta), r^{-}<$ $C_{6} h \varepsilon^{-1} \exp \left(-B_{1} h / \varepsilon\right), \mu-r^{-} / r^{+}>c_{7} \mu(\beta)$, and (3.10) then holds for (ii). For (iii) ( $c$ and $C$ are now fixed) and for $h$ sufficiently small: $r^{+}>C_{8}$; and $1-\mu>C_{9}(\beta)$ $>C_{10}(\beta) h / \varepsilon$. Let $\rho \equiv b_{j} h / \varepsilon$ and note that since $c<h / \varepsilon<C, r^{-}\left(\rho^{-}\right)=r^{-}(\rho)+$ $\left(\rho^{-}-\rho\right) D_{\rho} r^{-}(\xi)=r^{-}(\rho)+O(h)$, and similarly for $r^{+}$. Then $r^{-} / r^{+}=$ $r^{-}(\rho) / r^{+}(\rho)+O(h)=\exp \left(-b_{j} h / \varepsilon\right)+O(h)$, and hence $\mu(\beta)-r^{-} / r^{+}>c_{11} \mu$. The result (3.10) then follows.

3.3. Proof of Theorem 3.1. We will use Lemma 3.4 and separately estimate the error from using (1.5) to approximate $v(x) \equiv \exp (-b(0) x / \varepsilon)$ and $w(x)$. Starting first with $w(x)$, we denote the approximate solution by $W_{j}$ and the error $w\left(x_{j}\right)-W_{j}$ by $e_{j}$. If $k_{1}(h, \varepsilon) \geqslant 0$ and $k_{2}(h, \varepsilon) \geqslant 0$ are such that $R^{h}\left(k_{1} \varphi_{j}+k_{2} \psi_{j}\right)>R^{h}\left( \pm e_{j}\right)= \pm \tau_{j}$ for each $j=1, \ldots, J-1$, then the discrete maximum principle implies that $\left|e_{j}\right|<k_{1}\left|\varphi_{j}\right|+k_{2}\left|\psi_{j}\right|$ for each $j$, so we need to find suitable $k_{1}$ and $k_{2}$ and then verify (3.2). Consider first the case $h<\varepsilon$, and use (3.8) to evaluate $\tau_{j}=\tau_{j}(w)$, and use the standard form for the remainder terms in (3.8). Now $T^{2}$ and $T^{3}$ are uniformly $O\left(h^{2}\right)$ (i.e., bounded by $C h^{2}$ ) by Lemma 3.5 and

$$
\left|w^{(3)}\left(x_{j}\right)\right|<C+C \varepsilon^{-2} \exp \left(-\delta x_{j} / \varepsilon\right) \text {. }
$$

Thus, the contribution to the error from these terms satisfies (3.2a). For the remainder terms, we write out the treatment of the $r^{-} \varepsilon h^{-2} R_{3}\left(x_{j}, x_{j}-h, w\right)$ term; the treatment of the other remainder terms is very similar and so will not be reproduced. For $h<\varepsilon$, this term is bounded by $\left|C \varepsilon h^{-2} h^{4} w^{(4)}(\xi)\right|$ which is bounded by $C \varepsilon h^{2}\left(1+\varepsilon^{-3} \exp \left(-\delta x_{j-1} / \varepsilon\right)\right)$, and, observing that $c<\exp (-\delta h / \varepsilon)$ when $h<\varepsilon$, we see that this contribution again satisfies (3.2a). To consider $w$ in the case $h>\varepsilon$, we use (3.9) and the integral form for the remainder terms. We see that $\left|T^{2} w^{(2)}\left(x_{j}\right)\right|$ is bounded by $C h^{2}\left(1+\varepsilon^{-1} \exp \left(-\delta x_{j} / \varepsilon\right)\right)$, and, using the fact that for any given positive integer $k,(h / \varepsilon)^{k} \exp (-.5 \delta h / \varepsilon)<C$, and hence $\exp \left(-\delta x_{j} / \varepsilon\right)<$ $C(\varepsilon / h)^{k} \exp \left(-.5 \delta x_{j} / \varepsilon\right)$, one finds that the contribution to the error from this term satisfies (3.2b).

We will show explicitly how to deal with the remainder term $Y_{j} \equiv$ $-q^{-} b_{j-1} R_{1}\left(x_{j}, x_{j}-h, w_{x}\right)$ (the others being similar). This term (and only this term) requires a special treatment at $j=1$. For $j>1$, by (2.5) and (3.5b) and (3.7c),

$$
\begin{aligned}
\left|Y_{j}\right| & <C \varepsilon h^{-1} \int_{x_{j}-h}^{x_{j}} h\left|w^{(3)}(s)\right| d s<C \varepsilon \int_{x_{j}-h}^{x_{j}}\left(1+\varepsilon^{-2} \exp (-\delta s / \varepsilon)\right) d s \\
& <C \varepsilon\left(h+\varepsilon^{-2}(\varepsilon / \delta) \exp \left(-\delta x_{j-1} / \varepsilon\right)\right),
\end{aligned}
$$

which for $j>1$ is

$$
<C \varepsilon h+C \varepsilon h^{-1} \exp \left(-.5 \delta x_{j-1} / \varepsilon\right)
$$

and so, again, the addition to the error from this term satisfies (3.2b). In the last step in (3.12) we used the fact that $j>1$, so then $h \varepsilon^{-1} \exp \left(-.5 \delta x_{j-1} / \varepsilon\right)<C$. For 
$j=1$ this device is not available, and we observe that

$$
Y_{j}=-q^{-} b_{j-1}\left(R_{0}\left(x_{j}, x_{j}-h, w_{x}\right)+h w_{x x}\left(x_{j}\right)\right) \text {. }
$$

Then,

$$
\left|Y_{1}\right|<C \varepsilon h^{-1} \int_{0}^{h}\left(1+\varepsilon^{-1} \exp (-\delta s / \varepsilon)\right) d s+C \varepsilon\left(1+\varepsilon^{-1} \exp (-\delta h / \varepsilon)\right)
$$

so

$$
\left|Y_{1}\right|<C \varepsilon+C \varepsilon h^{-1},
$$

and, hence, it is true that

$$
\left|Y_{1}\right|<C \varepsilon h^{-1} \mu(\delta)^{j-1} \text { at } j=1 .
$$

Thus, (3.10c) shows that the term $Y_{1}$ leads to a term in the error estimate which satisfies (3.2b).

3.4. Proof of Theorem 3.1 Continued-Estimate for $v(x)$. We now show that the error from using (1.5) to approximate $v(x)=\exp (-b(0) x / \varepsilon)$ satisfies (3.2) which concludes the proof of Theorem 3.1. Let $b_{j}$ denote $b\left(x_{j}\right)$, and let $V_{j}, j=0, \ldots, J$, denote the approximation to $v(x)$ obtained via (1.5). Now

$$
\tau_{j}=R^{h}\left(e_{j}=v\left(x_{j}\right)-V_{j}\right)
$$

so

$$
\tau_{j}=R^{h} e_{j}=R^{h} v_{j}-Q\left(L v_{j}\right)
$$

and

$$
L v_{j}=\varepsilon v_{x x}\left(x_{j}\right)-b(x) v_{x}\left(x_{j}\right)=b_{0} \varepsilon^{-1}\left(b_{0}-b_{j}\right) v_{j} .
$$

The proof will use the fact that $\left(b_{0}-b_{j}\right)$ is small near the boundary layer at $x=0$.

We have $\tau_{j}(v)=\tau^{q}+\tau^{r}$, where

$$
\begin{gathered}
\tau^{q} \equiv-Q L v_{j}=\left\{-q^{-} b_{0} \varepsilon^{-1}\left(b_{0}-b_{j-1}\right) \exp \left(b_{0} h / \varepsilon\right)\right. \\
-\left(q^{-}+q^{+}\right) b_{0} \varepsilon^{-1}\left(b_{0}-b_{j}\right) \\
\left.-q^{+} b_{0} \varepsilon^{-1}\left(b_{0}-b_{j+1}\right) \exp \left(-b_{0} h / \varepsilon\right)\right\} v_{j} \\
\tau^{r} \equiv R^{h} v_{j}=\varepsilon h^{-2} v_{j}\left\{r^{-}\left(\rho^{-}\right)\left(\exp \left(b_{0} h / \varepsilon\right)-1\right)\right. \\
\left.+r^{+}\left(\rho^{+}\right)\left(\exp \left(-b_{0} h / \varepsilon\right)-1\right)\right\}
\end{gathered}
$$

and so

$$
\begin{aligned}
\tau^{r}=\varepsilon h^{-2} r^{+}\left(\rho^{+}\right) v_{j}\left\{\left(r^{-}\right)\left(\rho^{-}\right) / r^{+}\left(\rho^{+}\right)\left(\exp \left(b_{0} h / \varepsilon\right)-1\right)\right. & \left.+\exp \left(-b_{0} h / \varepsilon\right)-1\right\} .
\end{aligned}
$$

We first consider the case when $h \geqslant \varepsilon$. The following simple facts will be used: if $\xi$ is between $\rho^{-}$and $\rho^{+}$, then there is an $\eta$ between $b_{j-1}$ and $b_{j+1}$ such that $\xi=b(\eta) h / \varepsilon ;$ if $\alpha_{1}<\alpha_{2}$, then there is a point $p$ in $\left(\alpha_{1}, \alpha_{2}\right)$ such that $\exp \left(\alpha_{2}\right)-$ $\exp \left(\alpha_{1}\right)=\left(\alpha_{2}-\alpha_{1}\right) \exp (p)$; and, again, liberal use will be made of the fact that if $k$ is a positive integer and $c_{1}>0$ then there is a constant $C_{2}$, depending only on $c_{1}$ and $k$, for which $(x / \varepsilon)^{k} \exp \left(-c_{1} x / \varepsilon\right)<C_{2}$ for $x>0$. To bound $\tau^{r}$, we use (2.7) to observe that

$$
r^{-}\left(\rho^{-}\right)=r^{-}\left(\rho^{+}\right)+O\left(h^{3} \varepsilon^{-2} \exp (-b(\eta) h / \varepsilon)\right.
$$


and hence

$$
\begin{aligned}
r^{-}\left(\rho^{-}\right) / r^{+}\left(\rho^{+}\right)= & \exp \left(-\rho^{+}\right)+O\left(h^{2} \varepsilon^{-1} \exp (-b(\eta) h / \varepsilon)\right), \\
r^{-}\left(\rho^{-}\right) / r^{+}\left(\rho^{+}\right)= & \exp \left(-b_{0} h / \varepsilon\right)+\left[\exp \left(-\rho^{+}\right)-\exp \left(-b_{0} h / \varepsilon\right)\right] \\
& +O\left(h^{2} \varepsilon^{-1} \exp (-b(\eta) h / \varepsilon)\right) .
\end{aligned}
$$

The second term in (3.17c) is bounded by $O\left(x_{j} h \varepsilon^{-1} \exp \left(-b\left(p_{j}\right) h / \varepsilon\right)\right)$ for some $p_{j}$ in $\left(0, x_{j}\right)$. Now $\exp \left(-b\left(p_{j}\right) h / \varepsilon\right) \exp \left(b_{0} h / \varepsilon\right)<\exp \left(C h x_{j} / \varepsilon\right)$. Writing $v_{j}=$ $\exp ^{2}\left(-.5 b(0) x_{j} / \varepsilon\right)$, one of these factors can be used to bound the latter term for $h$ sufficiently small, and so (3.10b), (3.10c), and (3.17) lead to (3.2b) for the $\tau^{r}$ term.

For $\tau^{q}$, the last two terms from the right side of (3.16a) are bounded by $C x_{j} \varepsilon^{-1} v_{j}<C \varepsilon h^{-1} \exp \left(-.5 b(0) x_{j} / \varepsilon\right)$ which leads to (3.2b). For the first term on the right of (3.16a), the observation that $C x_{j-1} \varepsilon^{-1} \exp \left(b_{0} h / \varepsilon\right) v_{j}=C x_{j-1} \varepsilon^{-1} v_{j-1}$ suffices for $j>2$. For $j=1, b_{0}-b_{j-1}=0$ and the proof for $h>\varepsilon$ is complete.

We now commence the task of treating the error in $v(x)$ when $h<\varepsilon$. The overall approach is to Taylor expand everything in (3.16b) about $\rho \equiv b_{j} h / \varepsilon$. We have

$$
\begin{gathered}
r^{-}\left(\rho^{-}\right)=r^{-}(\rho)+\left(\rho^{-}-\rho\right) D_{\rho} r^{-}(\rho)+O\left(h^{4} / \varepsilon^{2}\right), \\
D_{\rho} r^{-}(\rho)=D_{\rho} r^{-}(0)+\rho D_{\rho}^{2} r^{-}(0)+O\left(h^{2} / \varepsilon^{2}\right),
\end{gathered}
$$

and so (throughout the rest of the paper terms which have been reduced to a nice form where (3.10) yields (3.2) will be generically denoted by $N$ )

$$
r^{-}\left(\rho^{-}\right)=r^{-}(\rho)-.5\left(\rho^{-}-\rho\right)+\rho\left(\rho^{-}-\rho\right) / 6+N
$$

from which one has

$$
\begin{aligned}
r^{-}\left(\rho^{-}\right)= & r^{-}(\rho)+h^{2} \varepsilon^{-1} b_{x}\left(x_{j}\right) / 4-h^{3} \varepsilon^{-1} b_{x x}\left(x_{j}\right) / 8 \\
& -h^{3} \varepsilon^{-2} b\left(x_{j}\right) b_{x}\left(x_{j}\right) / 12+N .
\end{aligned}
$$

Similarly,

$$
\begin{aligned}
r^{+}\left(\rho^{+}\right)= & r^{+}(\rho)+h^{2} \varepsilon^{-1} b_{x}\left(x_{j}\right) / 4+h^{3} \varepsilon^{-1} b_{x}\left(x_{j}\right) / 8 \\
& +h^{3} \varepsilon^{-2} b\left(x_{j}\right) b_{x}\left(x_{j}\right) / 12+N .
\end{aligned}
$$

Expanding $\exp \left(b_{0} h / \varepsilon\right)-1$ and $\exp \left(-b_{0} h / \varepsilon\right)-1$, we find, from (3.16b) and (3.19), that

$$
\tau^{r}=\varepsilon h^{-2} v_{j}\left\{r^{-}(\rho)\left(\exp \left(b_{0} h / \varepsilon\right)-1\right)+r^{+}(\rho)\left(\exp \left(-b_{0} h / \varepsilon\right)-1\right)\right\}+N
$$

and so

$$
\tau^{r}=\varepsilon h^{-2} v_{j} r^{+}(\rho)\left(\exp (-\rho)-\exp \left(-b_{0} h / \varepsilon\right)\right)\left(\exp \left(b_{0} h / \varepsilon\right)-1\right)+N
$$

Now observe that

$$
\begin{aligned}
Z_{j} & \equiv\left(\exp (-\rho)-\exp \left(-b_{0} h / \varepsilon\right)\right)\left(\exp \left(b_{0} h / \varepsilon\right)-1\right) \\
& =N+\left[-\left(b_{j}-b_{0}\right) h / \varepsilon+\left(b_{j}-b_{0}\right)^{2} h^{2} /\left(2 \varepsilon^{2}\right)\right]\left(b_{0} h / \varepsilon-\left(b_{0} h\right)^{2} /\left(2 \varepsilon^{2}\right)\right),
\end{aligned}
$$

and so

$$
\tau^{r}=\varepsilon h^{-2} v_{j}(1+\rho / 2) Z_{j}+N,
$$

from which, after considerable cancellation of terms, one finds that

$$
\tau^{r}=v_{j}\left(b_{0}-b_{j}\right) b_{0} / \varepsilon+N \text {. }
$$


The treatment of $\tau^{q}$ is fortunately not quite so tedious. Simply use (2.6) to expand $q^{-}$and $q^{+}$, expand $\left(b_{0}-b_{j+1}\right)$ and $\left(b_{0}-b_{j-1}\right)$ about $\left(b_{0}-b_{j}\right)$, expand $\exp \left(b_{0} h / \varepsilon\right)$ and $\exp \left(-b_{0} h / \varepsilon\right)$, and find that

$$
\tau^{q}=-v_{j}\left(b_{0}-b_{j}\right) b_{0} / \varepsilon+N
$$

Equations (3.22) and (3.23) combine to complete the proof of Theorem 3.1.

The final step in the proof of Theorem 1.1 is to show uniform $O\left(h^{2}\right)$ accuracy for the function $E(x) / b(x)$ from $(3.3 \mathrm{a}, \mathrm{b})$.

3.5. Treatment of $E(x) / b(x)$. Let $G(x) \equiv E(x) / b(x)$, and set $z(x)=1 / b(x)$. Then one can verify that

$$
L G=\varepsilon G_{x x}+b(x) G_{x}=\varepsilon E(x) z^{(2)}(x) .
$$

We write the truncation error for (1.5) applied to (3.24) as $\tau_{j}(G)=\tau^{r}+\tau^{q}=R^{h} G_{j}$ - $Q L G_{j}$, and we will prove that (1.5) gives a uniformly $O\left(h^{2}\right)$ accurate approximation to $G(x)$. Consider first the case $h>\varepsilon$. Then $\tau^{q}$ is immediately bounded by $C \varepsilon h \mu\left(B_{1}\right)^{j-1} / h$ which by $(3.10 \mathrm{c})$ leads to a contribution to the error estimate of $C \varepsilon h \mu^{j-1}$ which is certainly $O\left(h^{2}\right)$. It remains to deal with $\tau^{r}=R^{h} G_{j}$. The approach is to expand all terms about $x_{j}$ or $\rho \equiv b_{j} h / \varepsilon$. Terms which are directly seen to lead to uniformly $O\left(h^{2}\right)$ additions to the error are simply denoted by $N$. Define for $k<m$

$$
S(k, m) \equiv \exp \left\{-\varepsilon^{-1} \int_{x_{k}}^{x_{m}} b(\xi) d \xi\right\}
$$

Then

$$
\begin{aligned}
\tau^{r}=\varepsilon h^{-2} E_{j-1}\left\{r^{-} z_{j-1}+\left(-r^{-}-r^{+}\right) z_{j} S(j-1, j)\right. & \\
& \left.+r^{+} z_{j+1} S(j-1, j+1)\right\}
\end{aligned}
$$

and

$$
\begin{aligned}
& r^{-}\left(\rho^{-}\right)=r^{-}(\rho)+\left(\rho^{-}-\rho\right) D_{\rho} r^{-}(\rho)+N, \\
& z_{j+1}=z_{j}+h D_{x} z_{j}+N, \quad z_{j-1}=z_{j}-h D_{x} z_{j}+N, \\
& r^{+}\left(\rho^{+}\right)=r^{+}(\rho)+\left(\rho^{+}-\rho\right) D_{\rho} r^{+}(\rho)+N, \\
& -\varepsilon^{-1} \int_{x_{j-1}}^{x_{j}} b(s) d s=-\rho+b_{x}\left(x_{j}\right) h^{2} /(2 \varepsilon)+O\left(h^{3} / \varepsilon\right), \\
& -\varepsilon^{-1} \int_{x_{j-1}}^{x_{j+1}} b(s) d s=-2 \rho+O\left(h^{3} / \varepsilon\right) .
\end{aligned}
$$

Now for arbitrary $a$ and $\eta, \exp (a+\eta)=\exp (a)+\eta \cdot \exp (a)+.5 \eta^{2} \exp (\xi)$ for some $\xi$ between $a$ and $a+\eta$, from which we have

$$
\begin{aligned}
S(j-1, j) & =\exp (-\rho)+\exp (-\rho) b_{x}\left(x_{j}\right) h^{2} /(2 \varepsilon)+N, \\
S(j-1, j+1) & =\exp (-2 \rho)+N .
\end{aligned}
$$

From (3.25), (3.26) and (3.10) and the fact that

$$
r^{-}(\rho)(1-\exp (-\rho))+r^{+}(\rho)(\exp (-2 \rho)-\exp (-\rho)) \equiv 0,
$$


one verifies that

$$
\begin{aligned}
& \tau^{r}=\varepsilon h^{-2} E_{j-1}\left\{z_{j}\left(\rho^{-}-\rho\right) D_{\rho} r^{-}(\rho)-z_{x}\left(x_{j}\right) h r^{-}(\rho)\right. \\
& \quad-z_{j}\left(\rho^{-}-\rho\right) \exp (-\rho) D_{\rho} r^{-}(\rho)-z_{j}\left(\rho^{+}-\rho\right) \exp (-\rho) D_{\rho} r^{+}(\rho) \\
& -z_{j} r^{-}(\rho) \exp (-\rho) b_{x}\left(x_{j}\right) h^{2} /(2 \varepsilon)-z_{j} r^{+}(\rho) \exp (-\rho) b_{x}\left(x_{j}\right) h^{2} /(2 \varepsilon) \\
& \left.\quad+z_{j}\left(\rho^{+}-\rho\right) \exp (-2 \rho) D_{\rho} r^{+}(\rho)+z_{x}\left(x_{j}\right) h r^{+}(\rho) \exp (-2 \rho)\right\} .
\end{aligned}
$$

Now use the fact that $r^{-}(\rho)=\exp (-\rho) r^{+}(\rho)$ and (from Remark 2.5) $D_{\rho} r^{-}(\rho)=$ $D_{\rho} r^{+}(\rho)-1$ to eliminate $r^{-}$and $D_{\rho} r^{-}$from (3.27). Then replace $-b_{x}\left(x_{j}\right) z_{j}$ by $b_{j} z_{x}\left(x_{j}\right)$ in the two terms where it appears. Again use the latter equality to find that

$$
\begin{aligned}
& z_{j}\left(\rho^{-}-\rho\right)=z_{x}\left(x_{j}\right) \rho h / 2+O\left(h^{3} / \varepsilon\right), \\
& z_{j}\left(\rho^{+}-\rho\right)=-z_{x}\left(x_{j}\right) \rho h / 2+O\left(h^{3} / \varepsilon\right) .
\end{aligned}
$$

Using (3.28) in the expression for $\tau^{r}$ just obtained, one ascertains that $\tau^{r}$ has the form

$$
E_{j-1} \varepsilon h^{-2} z_{x}\left(x_{j}\right) h\{N+P(\rho)\}
$$

where $P(\rho)$ is a certain function of $\rho$. Using (2.5) and the common denominator $(1-\exp (-\rho))^{2}$, one can check that $P(\rho) \equiv 0$, and the result is in hand for $h>\varepsilon$.

The proof for the case $h \leqslant \varepsilon$ is very similar, but more Taylor expansion terms must be carried along, viz.,

$$
\begin{gathered}
r^{-}\left(\rho^{-}\right)=r^{-}(\rho)-b_{x}\left(x_{j}\right) h^{2} D_{\rho} r^{-}(\rho) /(2 \varepsilon) \\
+b_{x x}\left(x_{j}\right) h^{3} D_{\rho} r^{-}(\rho) /(4 \varepsilon)+N, \\
r^{+}\left(\rho^{+}\right)=r^{+}(\rho)+b_{x}\left(x_{j}\right) h^{2} D_{\rho} r^{+}(\rho) /(2 \varepsilon) \\
+b_{x x}\left(x_{j}\right) h^{3} D_{\rho} r^{+}(\rho) /(4 \varepsilon)+N, \\
q^{+}\left(\rho^{+}\right)=q^{+}(\rho)+N, q^{-}\left(\rho^{-}\right)=q^{-}(\rho)+N, \\
z_{x x}\left(x_{j-1}\right)=z_{x x}\left(x_{j}\right)-h z_{x x x}\left(x_{j}\right)+N, \\
z_{x x}\left(x_{j+1}\right)=z_{x x}\left(x_{j}\right)+h z_{x x x}\left(x_{j}\right)+N .
\end{gathered}
$$

Analogous to $(3.26 \mathrm{~d}-\mathrm{g})$, one has

$$
\begin{gathered}
S(j-1, j)=\exp (-\rho)\left\{1+h^{2} b_{x}\left(x_{j}\right) /(2 \varepsilon)-h^{3} b_{x x}\left(x_{j}\right) /(6 \varepsilon)\right\}+N, \\
S(j-1, j+1)=\exp (-2 \rho)\left\{1-h^{3} b_{x x}\left(x_{j}\right) /(3 \varepsilon)\right\}+N .
\end{gathered}
$$

Now $\tau^{r}$ is given by (3.25b), and $\tau^{q}$ is given by

$$
\begin{aligned}
\tau^{q}=-\varepsilon E_{j-1}\left\{z_{x x}\left(x_{j-1}\right) q^{-}\left(\rho^{-}\right)+z_{x x}(\right. & \left.x_{j}\right)\left(q^{-}\left(\rho^{-}\right)+q^{+}\left(\rho^{+}\right)\right) S(j-1, j) \\
& \left.+z_{x x}\left(x_{j+1}\right) q^{+}\left(\rho^{+}\right) S(j-1, j+1)\right\} .
\end{aligned}
$$

Also

$$
z_{x x}\left(x_{j}\right)=-2 b_{x}\left(x_{j}\right) z_{j} z_{x}\left(x_{j}\right)-z\left(x_{j}\right)^{2} b_{x x}\left(x_{j}\right)
$$


Now substitute (3.30) into (3.25b) and (3.31), and also expand $z_{j+1}$ and $z_{j-1}$ about $z_{j}$, and use (3.26h) and (3.32) to find that

$$
\begin{aligned}
\tau_{j}=\varepsilon h^{-2} E_{j-1}\{[ & -h z_{x}\left(x_{j}\right) r^{-}+.5 h^{2} z_{x x}\left(x_{j}\right) r^{-}-h^{3} z_{x x x}\left(x_{j}\right) r^{-} / 6 \\
& \left.+z_{x}\left(x_{j}\right) \rho h D_{\rho} r^{-} / 2-z_{x x}\left(x_{j}\right) \rho h^{2} D_{\rho} r^{-} / 4\right] \\
+ & {\left[\left(\exp (-\rho) D_{\rho} r^{-}-\exp (-\rho) D_{\rho} r^{+}-r^{-} \exp (-\rho)-r^{+} \exp (-\rho)\right)\right.} \\
& \cdot\left(b_{j} h^{2} /(2 \varepsilon)\right) b_{x}\left(x_{j}\right) / b\left(x_{j}\right)^{2} \\
+ & \left(-\exp (-\rho) D_{\rho} r^{-}-\exp (-\rho) D_{\rho} r^{+}+r^{-} \exp (-\rho) / 1.5\right. \\
+ & \left.+r^{+} \exp (-\rho) / 1.5\right) \\
+ & \left(b_{j} h^{2} /(2 \varepsilon)\right)\left(b_{x}\left(x_{j}\right) / b\left(x_{j}\right)^{2}\right) \exp (-2 \rho) D_{\rho} r^{+} \\
& +\left(\exp (-2 \rho) b_{\rho} r^{+}-4 r^{+} \exp (-2 \rho) / 3\right) \\
& \left.\cdot\left(b_{j} h^{3} /(4 \varepsilon)\right) b_{x x}\left(x_{j}\right) / b\left(x_{j}\right)^{2}\right] \\
& +r^{+} \exp (-2 \rho) h z_{x}\left(x_{j}\right)+\exp (-2 \rho)\left(b_{j} h^{3} /(4 \varepsilon)\right) \\
& \cdot 2 b_{x}\left(x_{j}\right) z_{x}\left(x_{j}\right)\left(1 / b_{j}\right) D_{\rho} r^{+} \\
\left.+r^{+} \exp (-2 \rho) h^{2} z_{x x}\left(x_{j}\right) / 2+r^{+} \exp (-2 \rho) h^{3} z_{x x x}\left(x_{j}\right) / 6\right] & \left.\left.+h^{3} z_{x x x}\left(x_{j}\right)\left(q^{-}-q^{+} \exp (-2 \rho)\right)\right]\right\} \\
+ & h^{2} z_{x x}\left(x_{j}\right)\left(-q^{-}-q^{-} \exp (-\rho)-q^{+} \exp (-\rho)-q^{+} \exp (-2 \rho)\right) \\
&
\end{aligned}
$$

$+N$

From (3.33), one can write $\tau_{j}$ in the form

$$
\begin{aligned}
\tau_{j}= & g_{1}(\rho) h z_{x}\left(x_{j}\right)+g_{2}(\rho) h^{2} z_{x x}\left(x_{j}\right)+g_{3}(\rho) h^{3} z_{x x x}\left(x_{j}\right) \\
& +g_{4}(\rho) b_{j} h^{3} \varepsilon^{-1} b_{x x}\left(x_{j}\right) z\left(x_{j}\right)^{2} / 4 .
\end{aligned}
$$

After replacing $r^{-}(\rho)$ by $\exp (-\rho) r^{+}(\rho)$ and $D_{\rho} r^{-}$by $D_{\rho} r^{+}-1$, it is quickly seen that $g_{1}(\rho)$ is exactly the function $P(\rho)$ in (3.29) which was found to vanish. Using (2.6) to expand $g_{2}(\rho)$ in the form $\alpha_{0}+\alpha_{1} \rho+N$, we find that $g_{2}(\rho)=0+N$ and, similarly, with $g_{3}$ and $g_{4}$, and the proof is complete.

IV. Accuracy of the Approximate Solution Between the Grid Points. Given problem (1.1) with $d \equiv 0$ and approximate solution $\left\{U_{j}\right\}$ obtained using (1.5), let $U(x)$ be defined by

$$
\begin{gathered}
\varepsilon U_{x x}+\bar{b} U_{x}=\bar{f} \text { for } x_{j-1}<x<x_{j}, \\
U\left(x_{j-1}\right)=U_{j-1}, \quad U\left(x_{j}\right)=U_{j}, \quad \bar{b}=\left(b_{j-1}+b_{j}\right) / 2, \\
\bar{f}=\left(f_{j-1}+f_{j}\right) / 2 \text { for } j=1, \ldots, J .
\end{gathered}
$$

The discussion in Section 2 demonstrates that $U(x)$ is in $C^{1}[0,1]$, and hence offers a potential approximation to $u(x)$ (the solution of (1.1)) between the grid points, as well as to $u_{x}$. The following result estimates the accuracy of this approximation. 
TheOREM 4.1. Let $U(x)$ be the function defined by (4.1) and let $u(x)$ be the solution of (1.1), where $d \equiv 0$ and $f$ is independent of $\varepsilon$. Assume $b$ and $f$ are in $C^{5}[0,1]$, and assume $b(x) \geqslant B_{1}$ on $[0,1]$ for some positive constant $B_{1}$. Then there are positive constants $C$ and $\sigma$, depending only on the set $S_{2}$ of Section 3 (with $m=5$ and $\left.B_{9}=\delta_{1}=0\right)$, such that

$$
\begin{gathered}
\max _{x_{j}<x<x_{j+1}}|U(x)-u(x)| \leqslant C h^{2}+C h^{3} \varepsilon^{-2} \exp \left(-\sigma x_{j} / \varepsilon\right) \\
\quad \text { for } h \leqslant \varepsilon, j=0, \ldots, J-1, \\
\max _{x_{j}<x<x_{j+1}}|U(x)-u(x)| \leqslant C h^{2}+C h \exp \left(-\sigma x_{j} / \varepsilon\right) \\
\text { for } h>\varepsilon, j=0, \ldots, J-1, \\
\varepsilon\left|U_{x}(0)-u_{x}(0)\right| \leqslant C h^{2} \varepsilon^{-1}+C h^{2}+C \varepsilon h \text { for } h<\varepsilon, \\
\varepsilon\left|U_{x}(0)-u_{x}(0)\right| \leqslant C h \text { for } \varepsilon \leqslant h .
\end{gathered}
$$

We note that the last term in (4.2c) is not "sharp." Indeed for $\varepsilon=1$, Pruess [12] has shown that $\left|U_{x}(x)-u_{x}(x)\right|$ is everywhere $O\left(h^{2}\right)$. Numerical experiments illustrating (4.2) were presented in [2].

Proof. The proof is obtained by observing that the error $e(x)=u(x)-U(x)$ on $\left(x_{j}, x_{j+1}\right)$ satisfies the differential equation

$$
M e \equiv \varepsilon e_{x x}+B e_{x}=(f-F)+(B-b) u_{x} \equiv g(x) \text { for } x_{j}<x<x_{j+1},
$$

where $B=\left(b_{j}+b_{j+1}\right) / 2$ and $F=\left(f_{j}+f_{j+1}\right) / 2$. Now Theorem 1.1 shows that $e\left(x_{j}\right)$ and $e\left(x_{j+1}\right)$ are $O\left(h^{2}\right)$. For the case $h \geqslant \varepsilon$ in $(4.2 \mathrm{~b})$, we use the comparison functions $\varphi(x) \equiv C h\left(x-x_{j}-2 h\right)$ and $\psi(x) \equiv-C h \cdot \exp (-\sigma x / \varepsilon)$, where $C$ and $\sigma$ are positive constants. Evaluating $M \varphi$ and $M \psi$, using Lemma 3.3 to bound $u_{x}$, picking $C$ sufficiently large and $\sigma$ sufficiently small, and applying the maximum principle for $M$ (e.g., [11, p. 6]), one obtains (4.2b). For the case $h<\varepsilon$, let $p$ denote the particular solution of (4.3) given by

$$
p(x)=B^{-1} \int_{x_{j}}^{x} g(s)[1-\exp (B(s-x) / \varepsilon)] d s .
$$

We will show directly that $p(x)$ satisfies (4.2a). Then $e(x)=p(x)+\rho(x)$, where $\rho(x)$ is a solution of $M \rho=0$, and $\rho\left(x_{j}\right)=e\left(x_{j}\right)=O\left(h^{2}\right)$ while $\rho\left(x_{j+1}\right)=O\left(h^{2}\right)-$ $p\left(x_{j+1}\right)$. By the maximum principle for $M, \rho$ is bounded by its values at $x_{j}$ and $x_{j+1}$, and thus (4.2a) holding for $p$ implies it is valid for $e$. To demonstrate (4.2a) for $p$ one can quickly reduce the situation to the case

$$
g(s)=\left[\left(x_{j}+.5 h-s\right) b_{x}\left(x_{j}+.5 h\right)+O\left(h^{2}\right)\right] u_{x}(s) .
$$

For $x_{j} \leqslant s \leqslant x \leqslant x_{j+1}, \exp (0)-\exp (B(s-x) / \varepsilon)$ is bounded by $B h / \varepsilon$, and using (4.4) and Lemma 3.3 then gives (4.2a).

For $(4.2 \mathrm{c}, \mathrm{d})$, observe that

$$
D_{x} p(x)=\varepsilon^{-1} \int_{x_{j}}^{x} g(s) \exp (B(s-x) / \varepsilon) d s .
$$

For $h \leqslant \varepsilon,\left|D_{x} p(x)\right|$ is bounded by $C \varepsilon^{-1}\left(h^{2}+h^{2} \varepsilon^{-1} \exp \left(-\sigma x_{j} / \varepsilon\right)\right)$ while for $h \geqslant \varepsilon$ it is bounded by $C \varepsilon^{-1}\left(h \varepsilon+h \cdot \exp \left(-\sigma x_{j} / \varepsilon\right)\right)$. Now,

$$
e(x)=p(x)+C_{1} \exp \left(-B\left(x-x_{j}\right) / \varepsilon\right)+C_{2}
$$


for some numbers $C_{1}$ and $C_{2}$. Using the fact that $e\left(x_{j}\right)$ and $e\left(x_{j+1}\right)$ are $O\left(h^{2}\right)$ and the previously obtained bounds for $p$, and "solving" for $C_{1}$, the estimates $(4.2 \mathrm{c}, \mathrm{d})$ are obtained.

V. A Numerical Experiment for the Case $d \neq 0$. Numerical experiments illustrating Theorem 1.1 were given in [3]. Here we present numerical results which suggest that, when $d \neq 0,(1.4)$ also achieves uniform second-order accuracy. The scheme (1.4) was used to obtain approximate solutions to the problem

$$
\varepsilon u_{x x}+b(x) u_{x}-d(x) u=f(x) \text { for } 0<x<1, u(0)=\alpha_{0}, u(1)=\alpha_{1},
$$

where $b(x)=(x+1)^{3}, d(x)=.31(x+1)^{5}, f(x)=-.43-.29 x-.23 x^{2}, \alpha_{0}=2.7$, and $\alpha_{1}=53 / 100$. For a given value of $\varepsilon$, the value of the exact solution $u$ at any grid point $x_{j}$ was taken to be the value at $x_{j}$ of the approximate solution of (5.1), obtained using (1.4) with $h=1 / 8192$. To obtain a wide variation of $h$ and $\varepsilon,(5.1)$ was solved with $\varepsilon \equiv h^{p}$ for various values of $p$. Uniform meshes with $h=1 / J$, $J=32,64, \ldots, 1024$, were used. The results are shown in Table 1 where $J$ is given in column 1, and where numerical results for a particular value of $p$ are given in each of the other columns. The $l^{\infty}$ error $\equiv$ maximum over $j=1, \ldots, J-1$ of $\left|u\left(x_{j}\right)-U_{j}\right|$ is listed under $E_{\infty}$. The numerical rate of convergence is determined from the $E_{\infty}$ values for two successive values of $J$ (e.g., $E_{\infty}^{1}$ and $E_{\infty}^{2}$ corresponding to $h=1 / J$ and $h=1 /(2 J)$, respectively) by

$$
\text { rate } \equiv\left(\ln E_{\infty}^{1}-\ln E_{\infty}^{2}\right) / \ln (2)
$$

The results seem to suggest uniform $O\left(h^{2}\right)$ accuracy.

TABLE 1

\begin{tabular}{|c|c|c|c|c|c|c|}
\hline & $\varepsilon=1$ & $\varepsilon=h^{.5}$ & $\varepsilon=h$ & $\varepsilon=h^{1.5}$ & $\varepsilon=h^{2}$ & $\varepsilon=h^{3}$ \\
\hline $\mathbf{J}$ & $E_{\infty}$ rate & $E_{\infty}$ rate & $E_{\infty}$ rate & $E_{\infty}$ rate & $E_{\infty}$ rate & $E_{\infty}$ rate \\
\hline \multirow[t]{2}{*}{32} & $4 \cdot 4 E-4$ & $6.9 E-4$ & 8. $6 E-4$ & 2. $6 \mathrm{E}-4$ & 2. $5 E-4$ & $2.6 \mathrm{E}-4$ \\
\hline & 2.00 & 1.90 & 1.92 & 2.03 & 1.94 & 1.95 \\
\hline \multirow[t]{2}{*}{64} & $1.1 E-4$ & 1. $9 E-4$ & $2.3 E-4$ & $6.3 E-5$ & $6.6 \mathrm{E}-5$ & $6.7 E-5$ \\
\hline & 2.00 & 1.91 & 1.96 & 1.97 & 1.97 & 1.98 \\
\hline \multirow[t]{2}{*}{128} & $2.7 \mathrm{E}-5$ & 4. $9 E-5$ & $5.8 E-5$ & $1.6 E-5$ & $1.7 \mathrm{E}-5$ & $1.7 E-5$ \\
\hline & 2.00 & 1.92 & 1.98 & 1.97 & 1.98 & 1.99 \\
\hline \multirow[t]{2}{*}{256} & $6.8 E-6$ & $1.3 E-5$ & $1.5 E-5$ & $4 \cdot 1 E-6$ & 4. $3 E-6$ & $4 \cdot 3 E-6$ \\
\hline & 1.98 & 1.94 & 1.99 & 1.98 & 2.00 & 2.00 \\
\hline \multirow[t]{2}{*}{512} & $1.7 E-6$ & $3.4 E-6$ & $3.7 E-6$ & $1.0 \mathrm{E}-6$ & $1.1 \mathrm{E}-6$ & $1.1 E-6$ \\
\hline & 1.93 & 1.96 & 2.01 & 2.00 & 2.01 & 2.01 \\
\hline 1024 & $4 \cdot 6 \mathrm{E}-7$ & 8. $7 E-7$ & $9.3 E-7$ & $2 \cdot 6 \mathrm{E}-7$ & $2.7 \mathrm{E}-7$ & $2.7 \mathrm{E}-7$ \\
\hline
\end{tabular}

Numerical results for (1.4) applied to (5.1) 
Applied Mathematics Branch-Code R44

Naval Surface Weapons Center

Silver Spring, Maryland 20910

Applied Mathematics Branch-Code R44

Naval Surface Weapons Center

Silver Spring, Maryland 20910

Mathematical Analysis Division

National Bureau of Standards

Washington, D.C. 20234

1. A. E. Berger, J. M. Solomon \& M. CIment, "Higher order accurate tridiagonal difference schemes for diffusion convection equations," Advances in Computer Methods for Partial Differential Equations-III (R. Vichnevetsky and R. S. Stepleman, Eds.), Proc. Third IMACS Conference on Computer Methods for Partial Differential Equations, June 1979, Lehigh University, pp. 322-330.

2. A. E. Berger, J. M. SOlomon \& M. CIMENT, Uniformly Accurate Difference Methods for a Singular Perturbation Problem, Proc. Internat. Conf. on Boundary and Interior Layers, Computational and Asymptotic Methods, June 3-6, 1980, Trinity College, Dublin, Ireland (J. J. H. Miller, Ed.), Boole Press, Dublin, 1980, pp. 14-28.

3. A. E. Berger, J. M. Solomon, M. Ciment, S. H. Leventhal \& B. C. Weinberg, "Generalized operator compact implicit schemes for boundary layer problems," Math. Comp., v. 35, 1980, pp. 695-731.

4. T. M. EL-Mistikawy \& M. J. WeRLE, "Numerical method for boundary layers with blowing-The exponential box scheme,” AIAA J., v. 16, 1978, pp. 749-751.

5. P. P. N. DE Groen \& P. W. Hemker, "Error bounds for exponentially fitted Galerkin methods applied to stiff two-point boundary value problems," Numerical Analysis of Singular Perturbation Problems (P. W. Hemker and J. J. H. Miller, Eds.), Academic Press, New York, 1979, pp. 217-249.

6. A. F. Hegarty, J. J. H. Miller \& E. O'RIORdan, Uniform Second Order Difference Schemes for Singular Perturbation Problems, Proc. Internat. Conf. on Boundary and Interior Layers, Computational and Asymptotic Methods, June 3-6, 1980, Trinity College, Dublin, Ireland (J. J. H. Miller, Ed.), Boole Press, Dublin, 1980, pp. 301-305.

7. A. M. IL'IN, "Differencing scheme for a differential equation with a small parameter affecting the highest derivative," Mat. Zametki, v. 6, 1969, pp. 237-248 = Math. Notes, v. 6, 1969, pp. 596-602.

8. R. B. Kellogg \& A. Tsan, "Analysis of some difference approximations for a singular perturbation problem without turning points," Math. Comp., v. 32, 1978, pp. 1025-1039.

9. J. LoRenZ, Stability and Consistency Analysis of Difference Methods for Singular Perturbation Problems, Proc. Conf. on Analytical and Numerical Approaches to Asymptotic Problems in Analysis, June 9-13, 1980, University of Nijmegen, The Netherlands (O. Axelsson, L. Frank and A. Van der Sluis, Eds.), North-Holland, Amsterdam, 1981.

10. J. J. H. MILLER, "Sufficient conditions for the convergence, uniformly in epsilon, of a three point difference scheme for a singular perturbation problem," Numerical Treatment of Differential Equations in Applications (R. Ansorge and W. Tornig, Eds.), Lecture Notes in Math., vol. 679, Springer-Verlag, Berlin and New York, 1978, pp. 85-91.

11. M. H. Protter \& H. P. Wennberger, Maximum Principles in Differential Equations, PrenticeHall, Englewood Cliffs, N.J., 1967.

12. S. A. Pruess, "Solving linear boundary value problems by approximating the coefficients," Math. Comp., v. 27, 1973, pp. 551-561.

13. M. E. Rose, "Weak-element approximations to elliptic differential equations," Numer. Math., v. 24, 1975, pp. 185-204.

14. D. R. SMrTH, "The multivariable method in singular perturbation analysis," SIAM Rev., v. 17, 1975, pp. 221-273. 